\title{
Peripapillary fluorescein angiographic findings in primary open angle glaucoma
}

\author{
Sei Yamazaki, Youichi Inoue, Keiji Yoshikawa
}

\begin{abstract}
Background-Detailed fluorescein angiographic findings in the disc circumference may be useful for evaluating the possible relation of the circumference to glaucomatous nerve damage.
\end{abstract}

Methods-Fluorescein angiograms of 25 eyes of 25 subjects with primary open angle glaucoma were observed after they had undertaken Octopus perimetry. Based on the retinotopic projection, disc sectors and corresponding visual field regions were set.

Results-Twenty three eyes (92\%) showed a zone of no fluorescence around the disc (non-fluorescent zone). Of these, the zone width of the 20 eyes that had visible ciliary vessels within the zone was wider than that of the other three eyes, and showed fluorescein diffusion from the outer boundary of the zone towards the disc. The diffusion reached the disc if the zone was narrow. In those 20 eyes, a standardised difference in the zone width of inferior temporal sector minus superior temporal sector correlated with the difference in mean loss of corresponding visual field regions $(r=0.48, p=0.0312)$.

Conclusion-The visible ciliary vessels suggest the absence of the choriocapillaris in the non-fluorescent zone, the width of which correlated with the visual field defect and may affect the amount of the fluorescein diffusion to the disc. This suggests that the diffusion might be related to optic nerve damage in glaucoma.

(Br F Ophthalmol 1996;80:812-817)

A number of studies ${ }^{1-13}$ demonstrated an association between the distribution of the peripapillary crescents and the localisation of optic nerve damage in glaucoma. Thus, the crescents may play some role in glaucomatous optic nerve damage. ${ }^{3514}$ Although the mechanism is unknown, there may be a contribution from some serum materials such as vasoconstrictors ${ }^{514}$ to the disc through the choriocapillaris surrounding it.

Fluorescein angiography, which can reveal vascularity and serum leakage, may give us more information about the peripapillary structure and serum dynamics. Though some investigators ${ }^{15-17}$ reported fluorescein angiographic findings for the peripapillary region in glaucoma, they referred to the global appearance of the choroidal filling in the posterior pole, not to the findings for the crescents or the region corresponding to them.
Baba and coworkers ${ }^{18}$ have noticed a phenomenon that the crescents (called conus in their paper) are not filled in fluorescein angiography. By means of this they graded the width of the crescents and then showed an association between the width of the temporal crescents and the central visual field loss in glaucomatous eyes. Despite this, that study ${ }^{18}$ did not deal with further details of fluorescein angiographic findings in the crescents apart from the phenomenon itself.

We studied fluorescein angiographic findings in the surroundings next to the disc in eyes with primary open angle glaucoma. We especially took note of a zone of no fluorescence around the disc (which may correspond to the crescent) and an association between the appearance of the zone and the type of visual field defect.

\section{Materials and methods}

Clinical records of 200 patients with the diagnosis of primary open angle glaucoma (POAG) were selected randomly by a computer search of the glaucoma patient database in the eye division of the Olympia Medical Clinic. Patients were diagnosed as having POAG if they showed intraocular pressure (IOP) of more than $21 \mathrm{~mm} \mathrm{Hg}$ with open angle on at least two different occasions, glaucomatous cupping and visual field changes, and no evidence of present or past history for ocular disease causing secondary IOP elevation. All patients were Japanese; we believe the cutoff value of $21 \mathrm{~mm} \mathrm{Hg}$ is high enough for the criterion of 'high' IOP to be given to Japanese patients $^{1920}$ who generally have a lower IOP than white people.

These patients were included in this study if they met the following criteria upon record review: consistency of glaucomatous disc cupping or nerve fibre layer defect with the visual field changes; no chorioretinal lesions that could contribute to visual field defect; no corneal opacity; no definite cataract or opacity in vitreous; no cataract surgery; availability of visual field tested by Octopus perimeter (PROGRAM 31); and availability of sharp fluorescein fundus angiogram (taken with Kowa RC-XV, Kowa Optimed Co Ltd, Tokyo, Japan). In addition to these, only the right eye was chosen for subjects with bilaterally affected eyes. Thus, finally 25 eyes of 25 subjects were included in this study.

For these subjects, we observed the fluorescein appearance in the disc circumference on series of angiograms of early venous phase 
A

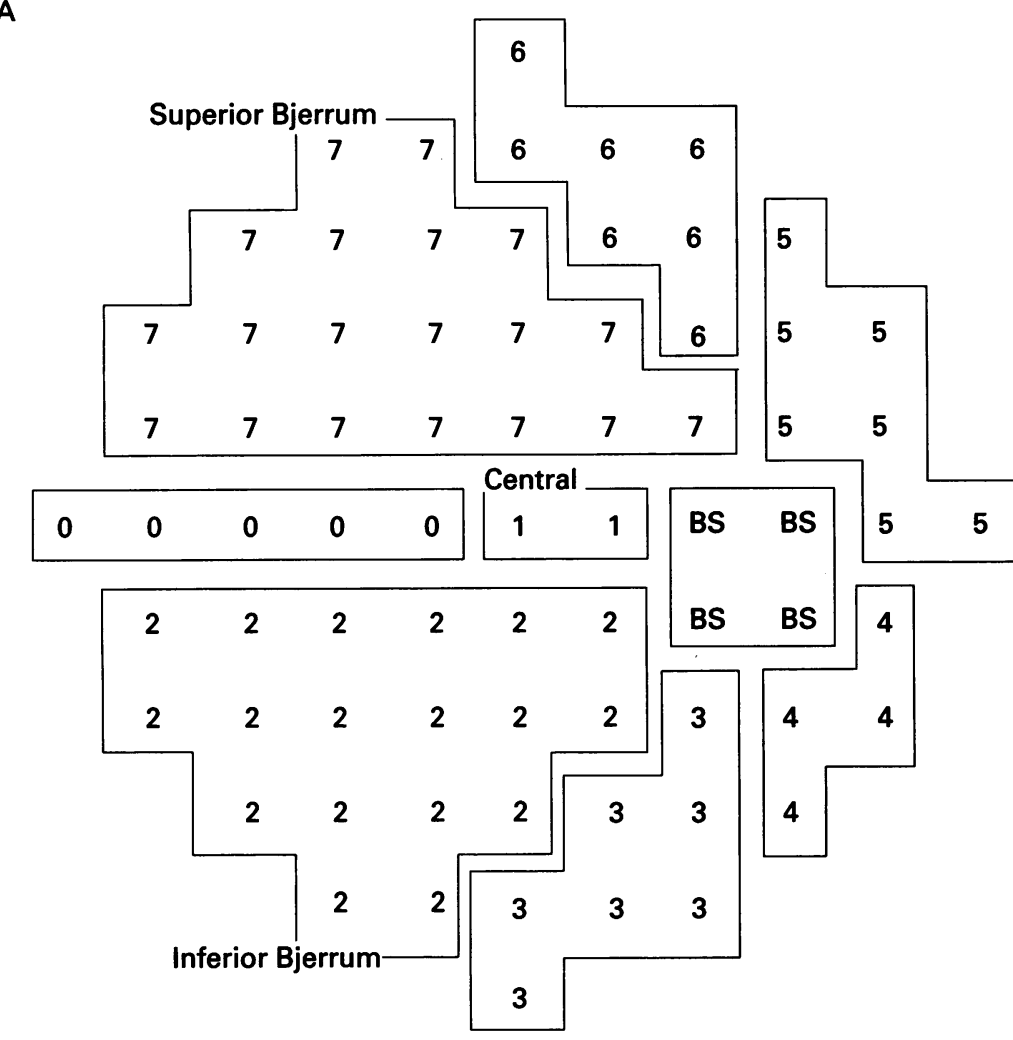

B

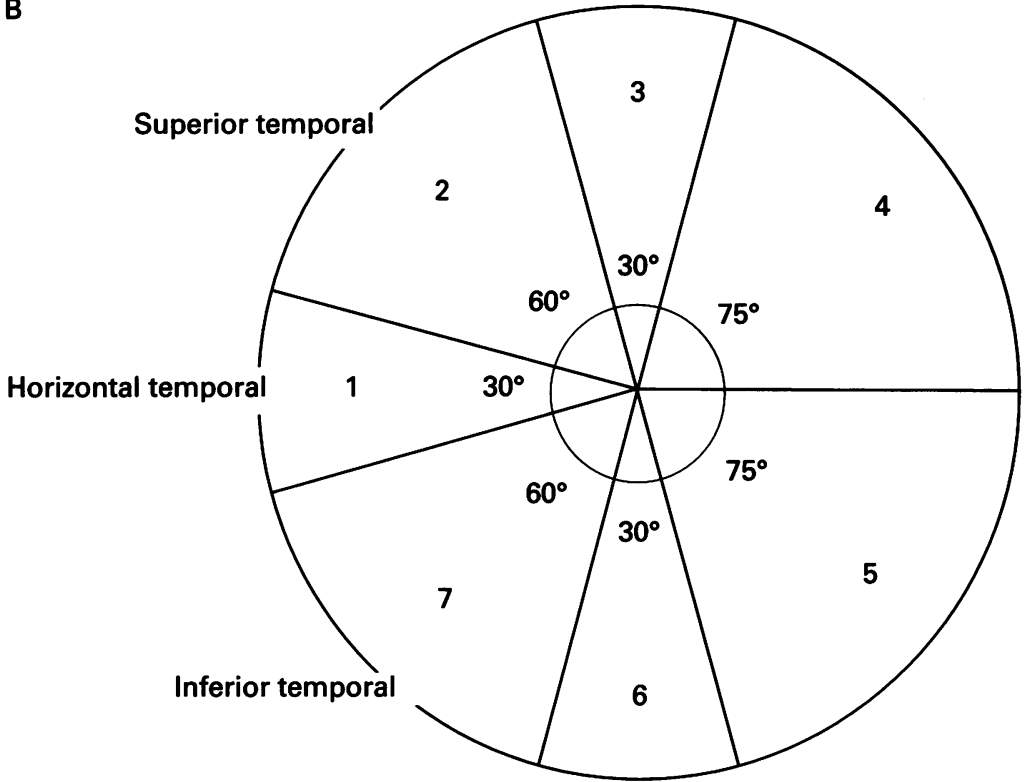

Figure 1 (A) Visual field regions defined in PROGRAM 31 (Octopus) for right eye. Each region corresponds to a sector defined with the same number presented in (B) except the five points numbered 0 , for which a corresponding sector was not set since they are on the border between region 2 and region 7 based on the retinotopic projection. (B) Sectors defined for the disc and the surrounding area for right eye. For the regions and sectors for left eye, mirror images were used.

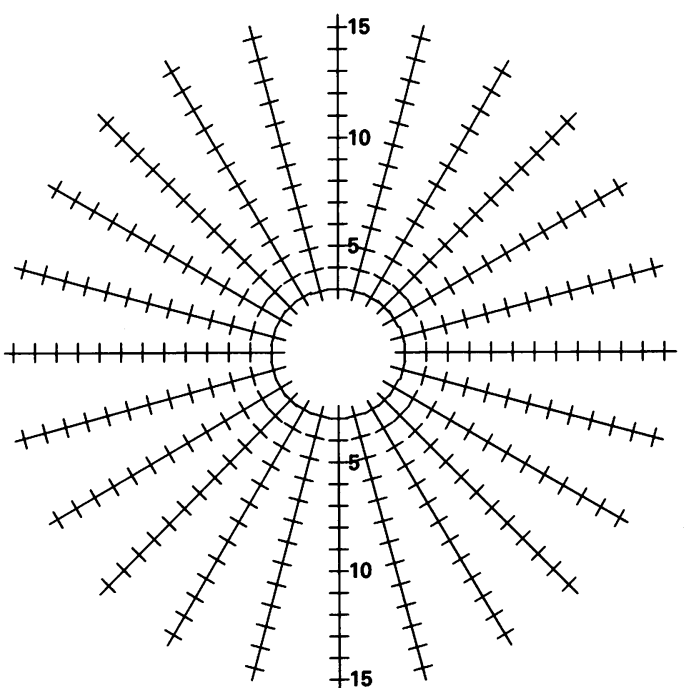

Figure 2 Grid used for the measurement of

non-fluorescent zone width. Radial bars are arranged 15 degrees apart from each other and scaled equally. Scales are arbitrary.

the visual field tested by Octopus (PROGRAM 31) were grouped into eight regions as shown in Figure 1A: superior Bjerrum region, central region, inferior Bjerrum region, and others. The disc and peripapillary area were divided into sectors, each sector corresponding to one visual field region, based on the retinotopic projection of visual field onto the $\operatorname{disc}^{21}$ : inferior temporal sector (corresponding to superior Bjerrum region), horizontal temporal sector (corresponding to central region), superior temporal sector (corresponding to inferior Bjerrum region), and others (Fig 1B). Mean loss $^{22}$ in each visual field region was calculated and used as the estimate for the extent of the optic nerve damage in the corresponding sector.

The fluorescein angiogram taken at the 20 degree setting was projected perpendicularly onto a grid designed for this study (Fig 2). With a modification of a previous method, ${ }^{7}$ the grid was divided by equally spaced radii, along which arbitrary distance units were established. The projection was set so that the scale reading of the superior pole of the disc on the upper vertical radius was the same as that of the inferior pole of the disc on the lower vertical radius. Then, three observers as a group traced onto the grid the disc margin and, if recognised, the outer boundaries of the nonfluorescent zone. The magnification rate of the projected angiogram was 20 times. We used the angiogram of early venous phase for the tracing since the width of the non-fluorescent zone generally became narrow with time. Between the measurements, each angiogram was masked to conceal the patient's clinical records.

The vertical diameter of the disc on the grid was measured and then the actual width of the non-fluorescent zone on each radius was calculated based on Bengtsson and Krakau's glass refraction method. ${ }^{23}$ Here, the calculated camera constant was 0.055 and we substituted 58.64 dioptres (Gullstrand) for the refractive power of the eye. Mean value of the actual non-fluorescent zone widths from all radii
Then we attempted to evaluate the association between the non-fluorescent zone width and visual field defect. To do this, visual field regions and disc sectors were set according to a modification of a previous method. ${ }^{21}$ Points in 

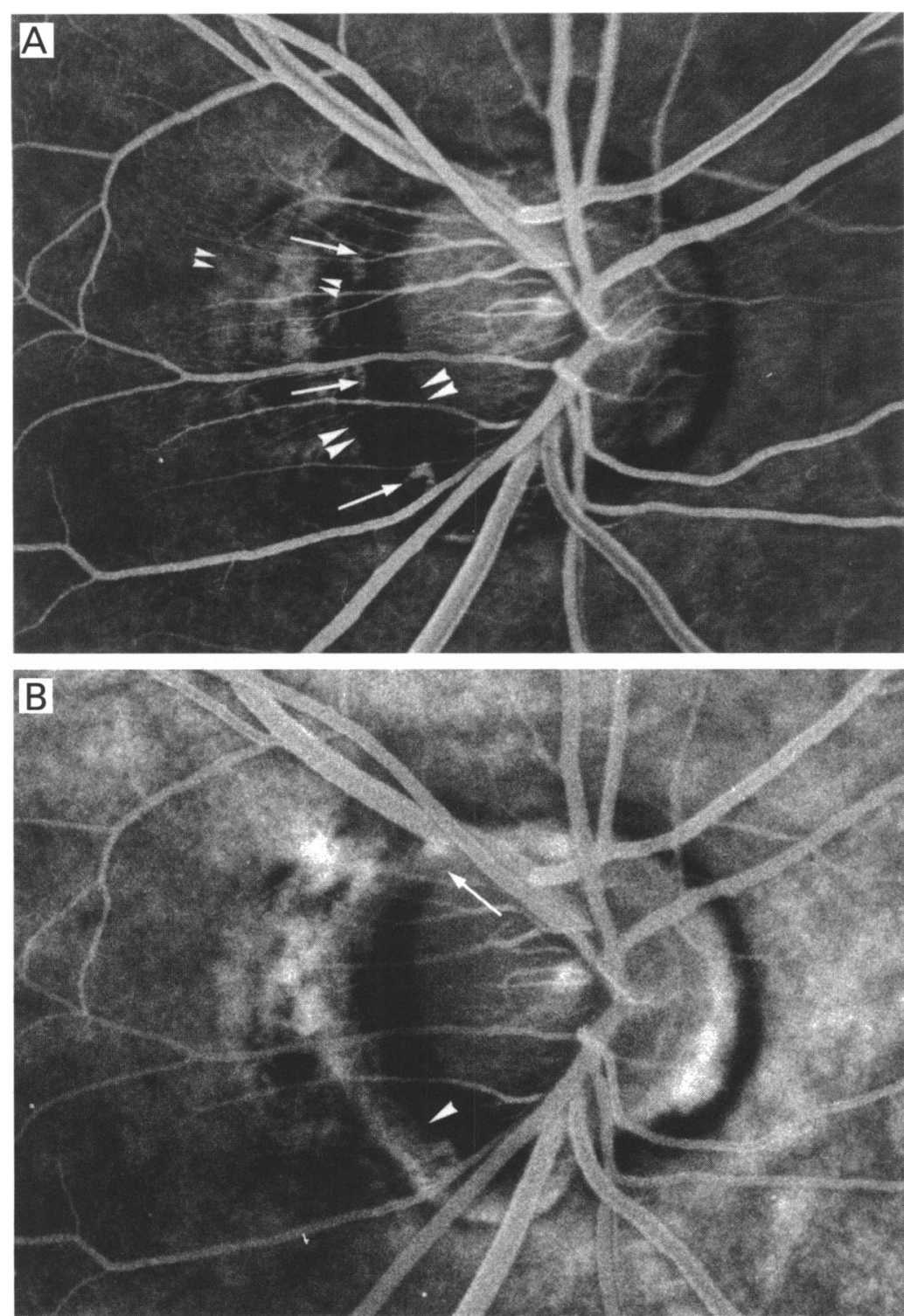

Figure 3 Fluorescein angiogram of the disc and peripapillary area (right eye). (A) Early phase (25 seconds). The non-fluorescent zone is shown between large arrowheads. Outside the temporal non-fluorescent zone, there is the irregularly fluorescent band (between small arrowheads). Arrows indicate the ciliary vessels within the non-fluorescent zone. A reduced fluorescein filling around the 7 o'clock position of the disc is seen in this case. (B) Late phase (302 seconds). Diffusing fluorescein from the periphery of the non-fluorescent zone is reaching the disc at the superior temporal sector (arrow), whereas in the inferior temporal sector, it is short of the disc edge (arrowhead). On the nasal side, the late hyperfluorescence is appearing along the disc margin.

Table 1 Visibility of ciliary vessels and non-fluorescent zone (NFZ) width

\begin{tabular}{llll}
\hline & \multicolumn{2}{l}{ Mean NFZ width $(S D)(\mathrm{mm})$} & \\
\cline { 2 - 3 } Sector of NFZ & $\begin{array}{l}\text { Ciliary vessels visible } \\
(n=20)\end{array}$ & $\begin{array}{l}\text { Ciliary vessels not visible } \\
(n=3)\end{array}$ & p Value \\
\hline Superior temporal & $0.34(0.17)$ & $0.16(0.05)$ & 0.0176 \\
Horizontal temporal & $0.52(0.21)$ & $0.20(0.09)$ & 0.0137 \\
Inferior temporal & $0.39(0.15)$ & $0.17(0.11)$ & 0.0358 \\
\hline
\end{tabular}

within a sector (Fig 1B), including radii on borders between sectors, was used as the estimate for the area size of non-fluorescent zone in the sector.

The Mann-Whitney U test was used to compare variables of different groups. Linear regression analysis was used to calculate correlation coefficients. The $F$ test was used for the test of statistical significance of the coefficients.

\section{Results}

The mean age of the 25 subjects was 54.4 years with a standard deviation (SD) of 12.6 years. Of the 25 subjects, 19 were male (age 55.6 (12.5) years) and six were female (age 50.5 (13.9) years). Refractive error distributed from -14.00 dioptres (D) to $+2.50 \mathrm{D}$ for all eyes $(-3.32(3.97) \mathrm{D})$. There was no statistically significant difference in age or refractive error between these 25 subjects and the 200 subjects selected first.

The non-fluorescent zone was simply defined as the zone of no fluorescence adjacent to the disc regardless of its shape or apparent width (Fig 3A). Outside the non-fluorescent zone in temporal sectors there generally was an irregularly fluorescent band. Of the 25 eyes, 23 eyes $(92 \%)$ showed a non-fluorescent zone. If present, it was generally the case that the nonfluorescent zone was apparently wider in temporal sectors than in nasal sectors, and that the boundaries were indistinct in nasal sectors. Because of this, measurement of the nonfluorescent zone width and analyses associated with the zone were carried out only for temporal sectors. Mean width of the non-fluorescent zone $(n=25)$ was $0.29(0.19) \mathrm{mm}$ for superior temporal sector, $0.44(0.25) \mathrm{mm}$ for horizontal temporal sector and $0.34(0.18) \mathrm{mm}$ for inferior temporal sector. (The width was defined as $0 \mathrm{~mm}$ if there was no nonfluorescent zone.)

It was also mostly the case that the ciliary vessels in the non-fluorescent zone were visible in temporal sectors whereas they were not in nasal sectors (Fig 3A). However, in three eyes of the 23 eyes having a non-fluorescent zone, the ciliary vessels were not visible even in temporal sectors, and the width of the zone for each temporal sector was apparently narrower in the three eyes than in the 20 eyes with visible ciliary vessels (Table 1 ).

Mean loss was $8.6(5.3) \mathrm{dB}(\mathrm{n}=25)$ for the whole tested visual field, 12.6 (8.8) $\mathrm{dB}$ for superior Bjerrum region, 3.1 (7.2) dB for central region, and 10.2 (7.9) dB for inferior Bjerrum region. The rate of false answers (number of pseudopositive and pseudonegative answers per number of pseudostimuli and the brightest stimuli) of the included visual fields was not more than $20 \%$.

Table 2 shows correlation coefficients of non-fluorescent zone width in each temporal sector with age, refractive error, and mean loss in the corresponding visual field region for all subjects. There was little correlation of age with non-fluorescent zone width. Refractive error showed a negative correlation with non-fluorescent zone width for each sector. Corresponding visual field loss showed a positive correlation with non-fluorescent zone width for the superior temporal sector only.

Fluorescein dynamics around the disc after the early venous phase seemed to vary in accordance with whether the non-fluorescent zone had visible ciliary vessels. If there was a non-fluorescent zone with visible ciliary vessels, hyperfluorescence appeared in the periphery of the zone after the early venous phase and it gradually diffused towards the disc. Finally in 
Table 2 Correlation coefficients of non-fluorescent zone (NFZ) width with age, refractive error, and mean loss in corresponding visual field region

\begin{tabular}{|c|c|c|c|c|c|c|}
\hline \multirow[b]{3}{*}{ Sector of NFZ } & \multicolumn{6}{|c|}{ Variables } \\
\hline & \multicolumn{2}{|l|}{ Age } & \multicolumn{2}{|c|}{ Refractive error } & \multicolumn{2}{|c|}{ Mean loss in corresponding region * } \\
\hline & $R$ & $p$ Value & $R$ & $p$ Value & $R$ & p Value \\
\hline Superior temporal & -0.05 & 0.8005 & -0.67 & 0.0003 & 0.47 & 0.0172 \\
\hline Horizontal temporal & -0.13 & 0.5311 & -0.51 & 0.0097 & 0.23 & 0.2695 \\
\hline Inferior temporal & -0.03 & 0.9063 & -0.47 & 0.0187 & 0.30 & 0.1485 \\
\hline
\end{tabular}

* Corresponding visual field region for each sector is shown in Figure 1. $\mathrm{n}=25$.

the late phase, the diffusing fluorescein had reached the disc edge in some sectors (Fig 3B). On the other hand, if there was no nonfluorescent zone with visible ciliary vessels (that is, there was a non-fluorescent zone but without visible ciliary vessels, or there was no apparent non-fluorescent zone), hyperfluorescence started to appear along the disc margin and then slightly expanded both to the inside and outside in the late phase (Fig 3B).

For the 20 eyes with visible ciliary vessels, we attempted to examine if the width of the non-fluorescent zone affected the diffusing fluorescein reaching the disc. Thus, three examiners decided independently whether the apex of the late fluorescein diffusion reached the disc edge or not in each temporal sector of the 20 eyes, and the majority of the three judgments was considered as our final decision for the site of the apex of the diffusion. This was done with each angiogram, masked to conceal the patient's clinical records. Table 3 summarises the association of site of the apex of the fluorescein diffusion with non-fluorescent zone width. Here, in each of the three temporal sectors, the non-fluorescent zone width was narrower in the group with the late fluorescein diffusion reaching the disc than the group without.

We evaluated whether the distribution of non-fluorescent zone spatially correlates with the pattern of visual field defect. Thus, the width difference of the inferior temporal sector minus the superior temporal sector was divided by the mean of the two for a standardisation. Then the standardised width difference was compared with the mean loss difference of superior Bjerrum region minus inferior Bjerrum region. The correlation was significant when only the 20 eyes with visible ciliary vessels were included $(r=0.48, p=0.0312$, $\mathrm{n}=20$, Fig 4). If the three eyes without visible ciliary vessels were added into the analysis, the correlation was weaker $(r=0.41, \mathrm{p}=0.0515$, $\mathrm{n}=23$ ).

Table 3 Site of apex of diffusing fluorescein and non-fluorescent zone (NFZ) width

\begin{tabular}{lllll}
\hline & \multicolumn{2}{l}{ Mean NFZ width $(S D)(m m)$} \\
\cline { 2 - 3 } & \multicolumn{2}{l}{ Apex of diffusing fluorescein } & \\
\cline { 2 - 4 } Sector of NFZ & \multicolumn{2}{l}{ Within NFZ } & At disc edge & p Value \\
\hline Superior temporal & $0.49(0.23)(\mathrm{n}=5)$ & $0.29(0.12)(\mathrm{n}=15)$ & 0.026 \\
Horizontal temporal & $0.62(0.15)(\mathrm{n}=14)$ & $0.28(0.08)(\mathrm{n}=6)$ & 0.0013 \\
Inferior temporal & $0.49(0.13)(\mathrm{n}=9)$ & $0.31(0.12)(\mathrm{n}=11)$ & 0.0088 \\
\hline
\end{tabular}

Only eyes showing ciliary vessels within non-fluorescent zone are included.

\section{Discussion}

A number of studies ${ }^{1-14}$ showed that the localisation of glaucomatous nerve damage correlates with the distribution of the peripapillary crescent. Our results showed that a similar relation exists by means of the non-fluorescent zone instead of the crescents seen in colour photographs. ${ }^{1-14}$ This may seem merely to indicate a fact that the non-fluorescent zone is equivalent to the crescents. However, there have been no reports describing the fluorescein angiographic findings in the disc circumference including the crescents or its association with glaucomatous visual field damage.

From our observations, there seemed to be two types of non-fluorescent zone. One is wide and is usually seen only in temporal sectors, has visible ciliary vessels, and shows late fluorescein diffusion within the zone. The other is narrow, is mostly seen in nasal sectors, does not have visible ciliary vessels, and does not show such fluorescein diffusion but accompanies late hyperfluorescence inside the zone (that is, on the disc margin). For convenience, we call the former the 'wide type' and the latter the 'narrow type'.

In the narrow type of non-fluorescent zone, the non-fluorescence may be due to intense pigmentation of retinal pigment epithelium (RPE) (double layered RPE, ${ }^{24}$ for example), which hides fluorescence of ciliary vessels. In the wide type, the visible ciliary vessels are probably a result of absence of the overlying $\mathrm{RPE}^{24}{ }^{25}$ If we think of RPE as modulating the choriocapillaris structure and function, ${ }^{26}$ the absence of the overlying PRE is enough for us

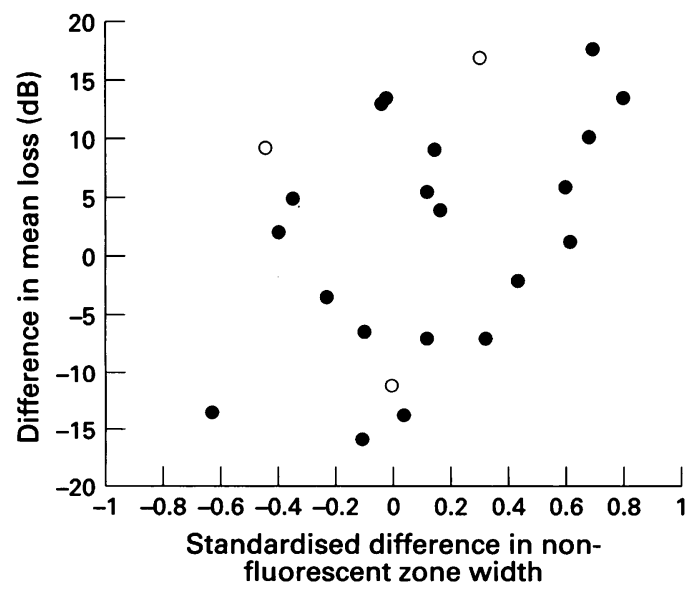

Figure 4 Scattergram for the standardised difference in non-fluorescent zone width and difference in mean loss. Subjects with visible ciliary vessels are shown as closed circles $(n=20)$ and those without as open circles $(n=3)$. 
to assume accompanying loss of the underlying choriocapillaris in the wide type of nonfluorescent zone. However, in the narrow type, the choriocapillaris is likely to extend immediately to the disc edge under the intensely pigmented RPE. Therefore, for the condition of peripapillary choriocapillaris, the narrow type of non-fluorescent zone may be the same as when there is no non-fluorescent zone.

Previous studies ${ }^{27-30}$ showed that the physiological diffusion of serum components occurs from the choroid to the disc. The RPE does not cover the lateral border of the choroid stopping around the disc, ${ }^{28}$ which allows the disc to receive extracapillary material from the choroid despite the fact that the capillaries of the disc possess tight junctions. ${ }^{28}{ }^{29}$ This phenomenon, together with the possible variations in peripapillary distribution of the choriocapillaris, can explain the observed variation in fluorescein dynamics in the late phase. Thus, the diffusing fluorescein within the wide type of non-fluorescent zone, and the late hyperfluorescence along the edge of the disc with the narrow type of non-fluorescent zone, or that without a non-fluorescent zone, probably represent the same serum diffusion, and the differences in those appearances may be due to how near the peripapillary choriocapillaris is to the disc edge.

In our observation, the diffusing fluorescein in the wide type of non-fluorescent zone tended to reach the disc if the zone width was short. This tendency further confirms the above explanation, and at the same time suggests that the width of the absence of the peripapillary choriocapillaris affects the diffusion reaching the disc. A further explanation of the spatial correlation between the visual field and non-fluorescent zone (Fig 4) is that the amount of the diffusion reaching the disc might be an essential factor in the correlation. Moreover, if we assume that the nonfluorescent zone had already existed before nerve damage, this might further indicate a possible role of diffusion in nerve damage.

Though some parts of the crescents could be the result of acquired changes, ${ }^{512}{ }^{14}$ recent studies $^{561213}$ showed that the change in the size of the crescents in glaucomatous eyes is minor and infrequent in the progression of glaucomatous nerve damage. In our observations, the correlation coefficient of non-fluorescent zone width with refractive error for each temporal sector was significant, whereas that with corresponding visual field loss was only significant for the superior temporal sector (Table 2). Although the findings of non-fluorescent zone we presented were those seen only in glaucomatous eyes, one frequently finds a nonfluorescent zone in eyes of myopic subjects who undertake fluorescein angiography for diseases other than glaucoma. Therefore, it may be likely that the non-fluorescent zone itself is mostly a feature of myopia and thus existed before the optic nerve damage. Further, we believe the correlation of nonfluorescent zone width with refractive error is a consistent finding with a correlation between myopia and crescents. ${ }^{31}$
It might be possible that serum diffusion to the disc plays a protective role against nerve damage in POAG: axons not able to receive enough serum diffusion from the peripapillary choroid might be more susceptible to a raised IOP. Thus, the late hyperfluorescence at the nasal edge of the disc, which was observed in most eyes in this study, could explain the phenomenon that nasal nerve fibres are relatively free of damage until the last stages of glaucoma. One might speculate on candidates for possible factors in serum diffusion. Nitric oxide, acting as a vasodilator when synthesised from endothelium, ${ }^{32}$ could be released from the peripapillary choroid and affect the sectoral circulation at the optic nerve. Scavengers for oxygen free radicals ${ }^{32}$ might also derive from the peripapillary choroid and could prevent some cytotoxic processes in the optic nerve to which oxygen free radicals might contribute.

Previous investigators ${ }^{1433}$ have already hypothesised that the influence of diffusion is associated with peripapillary crescents in glaucomatous eyes and supposed the diffusing substances were vasoconstrictors, which might worsen the disc environment. Thus, they hypothesised that the amount of diffusion might be greater in the region of a crescent, though this has not been proved. ${ }^{33}$ This may be opposite to our above interpretation about fluorescein dynamics in the peripapillary area.

It is important to speculate whether the high incidence of the non-fluorescent zone in POAG ( $80 \%$ for the wide type) is acceptable generally. In our results, distribution of refractive error was almost the same between the 200 subjects randomly selected and the 25 subjects finally included. This, together with the significant correlations between non-fluorescent zone width and myopic degree, suggests a general high incidence of non-fluorescent zone in POAG. Thus, an insufficiency in the serum diffusion to the disc in POAG might be a common phenomenon. We realise, however, that the cause-result relation between the amount of the diffusion and nerve damage requires further confirmation by longitudinal studies.

The method of grading the extent of fluorescein diffusion is a limitation of this study. If more quantitative methods than those used in our study are available, fluorescein dynamics might be better evaluated in their velocity or in the amount reaching the disc. This might be accomplished by a digital subtraction method for images of angiograms. Further studies are required to determine whether the correlation of non-fluorescent zone width with the amount of fluorescein diffusion is the same between eyes with and without glaucoma, or whether there are any factors that regulate the diffusing velocity or the diffusing amount to the disc.

We thank Professor Takashi Tokoro for his guidance in carrying out the research.

1 Primrose J. Early signs of the glaucomatous disc. $\mathrm{Br} \mathcal{F} O$ ph thalmol 1971:55:820-5.

2 Wilensky JT, Kolker AE. Peripapillary changes in glaucoma

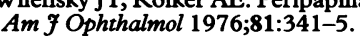


3 Anderson DR. Correlation of the peripapillary anatomy with the disc damage and field abnormalities in glaucoma. Doc Ophthalmol 1983;35:1-10.

4 Heijl A, Samander C. Peripapillary atrophy and glaucomatous visual field defects. Doc Ophthalmol 1985;42:403-7.

5 Nevarez J, Rockwood EJ, Anderson DR. The configuration of peripapillary tissue in unilateral glaucoma. Arch Ophthalmol 1988;106:901-3.

6 Rockwood EJ, Anderson DR. Acquired peripapillary changes and progression in glaucoma. Graefes Arch Clin Exp Ophthalmol 1988;226:510-5.

7 Buus DR, Anderson DR. Peripapillary crescents and halos in normal-tension glaucoma and ocular hypertension. Ophthalmology 1989;96:16-9.

8 Jonas JB, Nguyen XN, Gusek GC, Naumann GO. Parapapillary chorioretinal atrophy in normal and glaucoma eyes. I. Morphometric data. Invest Ophthalmol Vis Sci 1989;30. 908-18.

9 Jonas JB, Naumann GO. Parapapillary chorioretinal atrophy in normal and glaucoma eyes. II. Correlations. Invest Ophthalmol Vis Sci 1989;30:919-26.

10 Jonas JB, Gusek GC, Fernández MC. Correlation of the blind spot size to the area of the optic disc and blind spot size to the area of the optic disc and

11 Jonas JB, Fernández MC, Naumann GOH. Glaucomatous parapapillary atrophy. Arch Ophthalmol 1992;110:214-22.

12 Puska P, Raitta C. Peripapillary atrophy in unilateral capsular glaucoma. Graefes Arch Clin Exp Ophthalmol 1993;231 642-6.

13 Derick RJ, Pasquale LR, Pease ME, Quigley HA. A clinica study of peripapillary crescents of the optic disc in chronic experimental glaucoma in monkey eyes. Arch Ophthalmol 1994;112:846-50.

14 Anderson DR. Relationship of peripapillary haloes and crescents to glaucomatous cupping. In: Krieglstein GK, ed. Glaucoma update III. Berlin: Springer-Verlag, 1987:103-5.

15 Raitta C, Sarmela T. Fluorescein angiography of the optic disc and the peripapillary area in chronic glaucoma. Acta disc and the peripapillary a

16 Laatikainen L, Mäntyä P. Effects of a fall in the intraocular pressure level on the peripapillary fluorescein angiogram in chronic open-angle glaucoma. Acta Ophthalmol 1974;52 625-33.

17 Hitchings RA, Spaeth GL. Fluorescein angiography in chronic simple and low tension glaucoma. $\mathrm{Br} \mathcal{F}$ Ophthalmol 1977;61:126-32.
18 Baba $H$, Ochi $T$, Yoshikawa $\mathrm{K}$, Inoue $\mathrm{T}$, Inoue $\mathrm{Y}$. Implication of conus on central visual field in glaucoma. Fpn f Clin Ophthalmol (Rinsho Ganka) 1988;42:373-6.

19 Shiose Y, Kitazawa Y, Tsukahara S, Akamatsu T, Mizokam $\mathrm{K}$, Futa $\mathrm{R}$, et al. Epidemiology of glaucoma in Japan, a nationalwide glaucoma survey. Fpn f Ophthalmol 1991;35: 133-55.

20 Shiose Y. New perspectives on a nationalwide glaucoma survey in Japan. Fournal of the Eye (Atarashii Ganka) 1994; 11(Suppl 1):7-11.

21 Wirtschafter JD, Becker WL, Howe JB, Younge BR Glaucoma visual field analysis by computed profile of nerve fiber function in optic disc sectors. Ophthalmology 1982;89: 255-66.

22 Hirsch J. Statistical analysis in computerized perimetry. In: Whalen WR, Spaeth GL, eds. Computerized visual fields. Thorofare, NJ: Slack, 1985:326-32.

23 Bengtsson B, Krakau CET. Correction of optic disc measurements on fundus photographs. Graefes Arch Clin Exp Ophthalmol 1992;230:24-8.

24 Fantes FE, Anderson DR. Clinical histologic correlation of human peripapillary anatomy. Ophthalmology 1989;96:20-5.

25 Green WR. Retina. In: Spencer WH, ed. Ophthalmic pathology: an atlas and textbook. 3rd ed. Philadelphia: WB pathology: an atlas and

26 Korete GE, Reppucci V, Henkind P. RPE destruction causes choriocapillary atrophy. Invest Ophthalmol Vis Sci 1984;25: 1135-45.

27 Grayson MC, Laties AM. Ocular localization of sodium fluorescein. Arch Ophthalmol 1971;85:600-9.

28 Cohen AI. Is there a potential defect in the blood-retinal barrier at the choroidal level of the optic nerve canal? Invest Ophthalmol 1973;12:513-9.

29 Ben-Sira I, Riva CE. Fluorescein diffusion in the human optic disc. Invest Ophthalmol 1975;14:205-11.

30 Flage T. Permeability properties of the tissues in the optic nerve head region in the rabbit and the monkey. Acta Ophthalmol $1977 ; 55: 652-64$

31 Fulk GW, Goss DA, Christensen MT, Cline KB, HerrinLawson GA. Optic nerve crescents and refractive error. Optometry And Vision Science 1992;69:208-213.

32 Schumer RA, Podos SM. The nerve of glaucoma! Arch Ophthalmol 1994;112:37-44.

33 Rader J, Feuer WJ, Anderson DR. Peripapillary vasoconstriction in the glaucomas and the anterior ischemic optic neuropathies. Am f Ophthalmol 1994;117:72-80. 